\title{
Condições assintóticas de contorno em osciladores harmônicos e aplicações
}

\author{
Asymptotic boundary conditions in harmonic oscillators and applications \\ Adriano Doff*10 \\ ${ }^{1}$ Universidade Tecnológica Federal do Paraná, 84016-210, Ponta Grossa, PR, Brasil
}

Received on January 06, 2020. Revised on February 10, 2020. Accepted on February 24, 2020.

\begin{abstract}
Neste trabalho apresentamos uma análise do comportamento assintótico de osciladores harmônicos amortecidos e forçados considerado a representação no espaço $\left(x^{2}, \dot{x}^{2}\right)$. Verificamos então que a partir da análise do comportamento assintótico das condições de contorno, tomando como exemplo o caso particular de osciladores harmônicos amortecidos e forçados, é possível inferir o comportamento dinâmico nesta região para sistemas mais complexos, como oscilador não-linear descrito na seção 5. Algumas implicações da solução assintótica para este sistema são discutidas ao fim desta seção, entretanto, uma análise mais detalhada em relação ao comportamento analítico deste sistema sairia do escopo deste trabalho .

Palavras-chave: Osciladores harmônicos Amortecidos e forçados, Osciladores não-lineres, Condições de contorno
\end{abstract}

In this paper we present an analysis of the asymptotic behavior of damped and forced harmonic oscillators considering the $\left(x^{2}, \dot{x}^{2}\right)$ representation, we find that from the analysis of the asymptotic behavior of boundary conditions, taking as an example the case of damped and forced harmonic oscillators, which its possible infer the dynamical behavior in this region for more complex systems, such as the nonlinear oscillator described in section 5.Some implications of the asymptotic solution for this system are discussed at the end of this section. However, a more detailed analysis of the analytical behavior of this system would be beyond the scope of this paper.

Keywords: Damped and forced harmonic oscillators, Nonlinear Oscillators, Boundary Conditions.

\section{Introdução}

Fenômenos oscilatórios são de extremo interesse em todos os campos da física 1,2 e o tópico associado a oscilações em particular é um assunto visto na maioria dos cursos introdutórios de física básica. Tradicionalmente, após a introdução dos conceitos de oscilador harmônico e oscilador harmônico amortecido, passa-se então a análise do oscilador harmônico amortecido e forçado [3 5]. A título de exemplificação de sistemas físicos que são governados por uma equação do tipo oscilador harmônico amortecido e forçado, o circuito RLC submetido a uma tensão periódica usualmente é o exemplo considerado. Neste trabalho apresentamos uma análise do comportamento assintótico de osciladores harmônicos amortecidos e forçados considerado a representação no espaço $\left(x^{2}, \dot{x}^{2}\right)$, a abordagem assumida em tal plano mapeia o comportamento da energia total para uma partícula sujeita a um potencial na forma $a x^{2}(t)$, e como verificaremos permite a avaliação mesmo para sistemas não-lineares da evolução dinâmica de $x(t), \dot{x}(t)$ de forma independente. Neste ponto devemos salientar que o termo comportamento assintótico corresponde a descrição do comportamento

*Endereço de correspondência: agomes@utfpr.edu.br do sistema físico na região assintótica do tempo, ou seja para $(t \rightarrow \infty)$.

Esta forma particular de representação não é usualmente empregada na literatura, porém, como discutimos ao longo do texto consiste de uma ferramenta útil e que pode ser empregada como uma análise complementar a descrição de uma variedade de sistemas dinâmicos. Sendo a equação responsável pela dinâmica do oscilador harmônico amortecido e forçado uma equação diferencial de segunda ordem, na maioria dos textos o tratamento desta é feito considerando as técnicas usuais do cálculo diferencial e integral. Entretanto, neste trabalho decidimos em optar por uma abordagem um pouco diferente que foi apresentada na Ref. [6], assim, a seção 2 foi incluida a fim de ilustrar o comportamento de um oscilador harmônico amortecido na presença de uma força externa considerando a aplicação e exemplificação desta. Na seção 3 apresentamos uma breve discussão de como o comportamento assintótico das condições de contorno para o sistema oscilador harmônico amortecido no espaço $\left(x^{2}, \dot{x}^{2}\right)$ pode ser empregado para inferir o comportamento dinâmico na região assintótica. Na seção 4, extendemos os resultados da seção anterior para o caso do oscilador harmônico amortecido e forçado para um forçamento periódico. Na seção 5 verificamos que análise do comportamento assintótico das condições de contorno 
, tomando como exemplo o caso particular de osciladores harmônicos amortecidos e forçados, permite inferir o comportamento dinâmico nesta região para sistemas mais complexos, como oscilador não-linear descrito na seção 5 . Na seção 6 apresentamos nossas conclusões.

\section{O método de operadores aplicado ao oscilador harmônico amortecido e forçado}

Como comentamos fenômenos oscilatórios são de extremo interesse em todos os campos da física, bem como em diversas áreas de engenharia. Tradicionalmente o tópico associado a oscilações é um assunto visto na maior parte dos cursos introdutórios de física básica. Abaixo descrevemos a equação de movimento para um oscilador harmônico amortecido sujeito a uma força externa dependente do tempo que denotamos por $F(t)$, já devidamente parametrizado

$$
\ddot{x}+2 \gamma \dot{x}+\omega_{0}^{2} x=\frac{F(t)}{m}
$$

onde identificamos $\gamma=\frac{b}{m}, \omega_{0}^{2}=\frac{k}{m}$, sendo $\omega_{0}$ a frequência natural de oscilação do sistema e $b$ o coeficiente de amortecimento. A técnica que descrevemos nesta seção foi apresentada na Ref. [6], apenas iremos considerar um pouco mais de detalhe tendo em vista a sua aplicação por parte de estudantes dos cursos de engenharia. Introduzindo os operadores diferenciais

$$
\dot{x}=\frac{d x(t)}{d t}=D x, \ddot{x}=\frac{d x(t)}{d t}=D^{2} x
$$

podemos escrever a Eq.(1) na forma

$$
\left(D^{2}+2 \gamma D+\omega_{0}^{2}\right) x(t)=\frac{F(t)}{m}
$$

sendo agora $D$ tratado como um operador, assim

$$
\left(D-P_{+}\right)\left(D-P_{-}\right)=\frac{F(t)}{m}
$$

onde $P_{+}$e $P_{-}$são constantes a serem determinadas. Uma vez que

$$
\begin{aligned}
& \left(D-P_{+}\right)\left(D-P_{-}\right) x(t)= \\
& \quad=\ddot{x}-\left(P_{+}+P_{-}\right) \dot{x}+x P_{+} P_{-}=\frac{F(t)}{m}
\end{aligned}
$$

a comparação desta expressão com a Eq.(1) leva a identificação

$$
\begin{aligned}
& P_{+}+P_{-}=-2 \gamma \\
& P_{+} P_{-}=\omega_{0}^{2} .
\end{aligned}
$$

O sistema de equações acima corresponde a

$$
P_{ \pm}^{2}+2 \gamma P_{ \pm}+\omega_{0}^{2}=0
$$

cuja a solução leva

$$
P_{ \pm}=-\gamma \pm \sqrt{\gamma^{2}-\omega_{0}^{2}}
$$

A solução geral em termos da equação operatorial pode ser encontrada considerando

$$
Z_{ \pm}(t)=\left(D-P_{ \pm}\right) x(t)=\dot{x}-P_{ \pm} x
$$

que implica em

$$
\dot{Z}_{ \pm}(t)-P_{ \pm} Z_{ \pm}(t)=\frac{F(t)}{m}
$$

cuja a solução geral leva a

$$
Z_{\mp}(t)=e^{P_{ \pm} t}\left[\int_{0}^{t} e^{-P_{ \pm} t} \frac{F(t)}{m} d t+C_{\mp}\right] .
$$

Na expressão acima $C_{\mp}$ é uma constante de integração a ser determinada e seu valor irá depender das condições contorno consideradas, da Eq.(9), ganhamos

$$
\begin{aligned}
& Z_{-}(t)=\dot{x}(t)-P_{-} x(t) \\
& Z_{+}(t)=\dot{x}(t)-P_{+} x(t),
\end{aligned}
$$

que nos permite escrever após alguma algebra

$$
\begin{aligned}
& x(t)=\frac{Z_{-}(t)-Z_{+}(t)}{\left(P_{+}-P_{-}\right)} \\
& \dot{x}(t)=\frac{Z_{-}(t) P_{+}}{\left(P_{+}-P_{-}\right)}-\frac{Z_{+}(t) P_{-}}{\left(P_{+}-P_{-}\right)} .
\end{aligned}
$$

\section{A energia total e as condições de contorno no assintótico do OHS e OHS amortecido no plano $\left(x^{2}, \dot{x}^{2}\right)$}

Nesta seção definiremos o OHS(oscilador Harmônico Simples) no plano $\left(x^{2}(t), \dot{x}^{2}(t)\right)$, a energia total para este sistema conservativo é representada por

$$
E_{T}=\frac{1}{2} m \dot{x}^{2}(t)+\frac{1}{2} k x^{2}(t)
$$

a partir desta, obtemos

$$
\frac{d E_{T}}{d t}=\dot{x}(t)\left(\ddot{x}(t)+\omega_{0}^{2} x(t)\right)=0
$$

onde $\omega_{0}^{2}=\frac{k}{m}$ é frequência caracteristica, e a equação acima caracteriza a equação de movimento para um sistema conservativo, o OHS. Considerando ainda a Eq. (14) , temos

$$
\frac{2 E_{T}}{m}=\frac{2 E_{c}}{m}+\frac{2 E_{p}}{m}=\dot{x}^{2}(t)+\omega_{0}^{2} x^{2}(t) .
$$

A partir da expressão acima, podemos escrever

$$
\frac{\frac{2 E_{c}}{m}}{\frac{2 E_{p}}{m}}=\frac{\dot{x}^{2}(t)}{\omega_{0}^{2} x^{2}(t)},
$$


então, representar no plano $\left(E_{p}, E_{c}\right)=\left(x^{2}, \dot{x}^{2}\right)$ a equação paramétrica

$$
\dot{x}^{2}(t)=\omega_{0}^{2}\left(\frac{E_{c}}{E_{p}}\right) x^{2}(t)
$$

que corresponde a uma reta parametrizada por

$$
z(t)=\dot{x}^{2}=a x^{2}=a y(t)
$$

onde o coeficiente linear é $b=0$, e o coefiente angular é dado por

$$
a=\omega_{0}^{2}\left(\frac{E_{c}}{E_{p}}\right) .
$$

A solução geral para a Eq. 15, pode ser expressa por

$$
x(t)=c_{1} \cos \left(\omega_{0} t\right)+c_{2} \operatorname{sen}\left(\omega_{0} t\right) .
$$

Para a escolha apropriada de constantes é possível verificar que para um sistema conservativo que o coeficiente angular $a=\omega_{0}^{2}\left(\frac{E_{c}}{E_{p}}\right)=\omega_{0}^{2}$. A partir da solução numérica da Eq. 15], para cada instante de tempo t, a linha azul na Figura 11 reflete o comportamento dinâmico para a energia total do sistema, de forma que sempre temos sobre a linha para qualquer $t$ a conservação de $\frac{2 E_{T}}{m}=\dot{x}^{2}(t)+\omega_{0}^{2} x^{2}(t)=1$ para a escolha de unidades dimensionalmente coerentes, onde consideramos a partir deste ponto $\omega_{0}^{2}=1$.

Assim, obtemos $\dot{x}(t)=0(t=2 \pi n), \frac{2 E_{p}(t)}{m}=x^{2}(t)=$ $x^{2}(0)=1$, da mesma forma que para $x(t)=0\left(t=\frac{\pi}{2} n\right)$, $\frac{2 E_{c}(t)}{m}=\dot{x}^{2}(t)=1$, e a linha em azul representa para

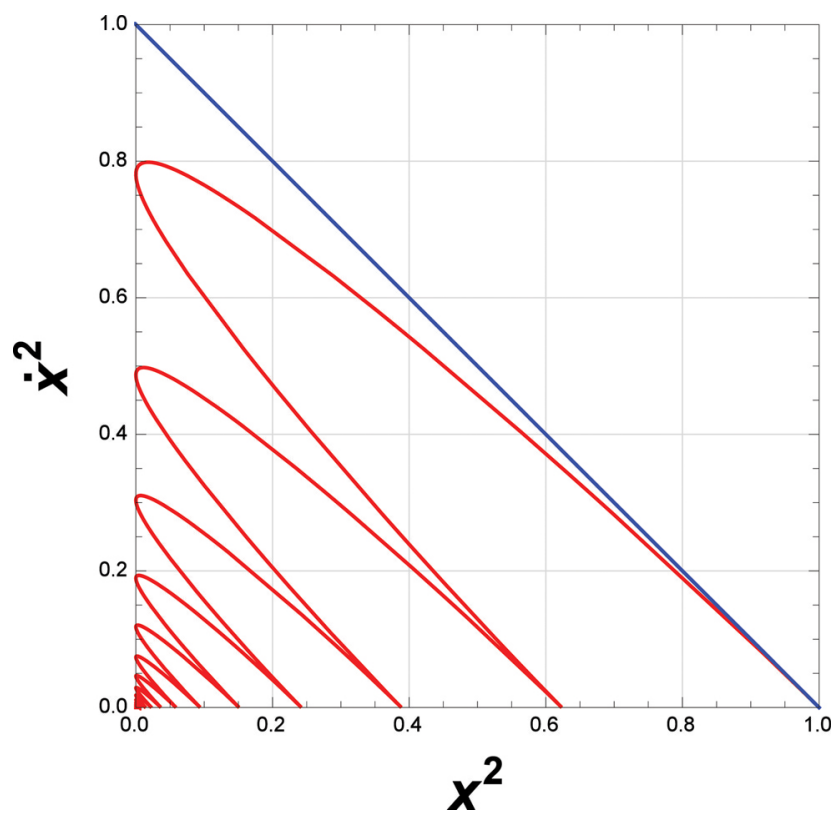

Figura 1: Comportamento no plano $\left(x^{2}, \dot{x}^{2}\right)$ para o OHS e OHS amortecido.

${ }^{1}$ Todas as Figuras do presente trabalho foram geradas empregando o software Mathematica algum t sempre o conjunto de condições de contorno a ser satisfeita,

$$
\frac{2 E_{T}(t)}{m}=\dot{x}^{2}(t)+x^{2}(t)=1 .
$$

Então, podemos dizer que a linha continua azul reflete nas condições de contorno na região assintótica o comportamento esperado para um sistema conservativo, onde a energia total disponível pelo sistema se mantem fixa no plano $\left(x^{2}, \dot{x}^{2}\right)$, assumindo $t \rightarrow \infty$. Para um sistema dissipativo, onde por exemplo $2 \gamma=0.15$, temos o comportamento do OHS amortecido descrito pela linha continua em vermelho. Este resultado descrito na Figura 1 agora corresponde a solução numérica da Eq.(1) no plano $\left(x^{2}, \dot{x}^{2}\right)$, para $\mathrm{F}(\mathrm{t})=0$. Neste caso é possível mostrar que a solução geral corresponde a

$$
x(t)=e^{-\gamma t}\left(c_{1} \cos (\omega t)+c_{2} \operatorname{sen}(\omega t)\right) .
$$

É possível redefinir uma nova amplitude que decresce com o coeficiente de atrito, $A_{\gamma}(t) \approx x(0) e^{-\gamma t}$, de forma que a partir da comparação entre as soluções com $\gamma=0$, e a equação acima permite que a Eq. 19 neste caso seja re-escrita na forma

$$
a \approx \omega^{2} e^{-\gamma t}
$$

E podemos, então considerar na região assintótica

$$
x(t) \approx e^{-\gamma t} \cos (\omega t) .
$$

Como a Figura 1(linha vermelha) ilustra, agora temos para cada ciclo(nT) retas com um coeficiente angular que decrescem com t. A aplicabilidade desta forma de representação é que o comportamento acima pode ser diretamente lido a partir da Figura 1, uma vez que para $\gamma<<1\left(\gamma=\frac{0.15}{2}\right)$

$$
e^{-\gamma t} \approx\left(1-\gamma t+\frac{\gamma^{2} t^{2}}{2}-\frac{\gamma^{3} t^{3}}{6}+O\left(\gamma^{4}\right) \ldots\right),
$$

e para os valores lidos diretamente no eixo-x da Figura 1(linha vermelha)

$$
\begin{aligned}
& t=2 \pi, e^{-\gamma t} \approx 0.624 \\
& t=4 \pi, e^{-\gamma t} \approx 0.389 \\
& t=6 \pi, e^{-\gamma t} \approx 0.234
\end{aligned}
$$

é possível identificar no eixo x da Figura 1 o comportamento oscilatório de $\left(E_{T}(t)\right)$, justamente na forma $e^{-\gamma t}$ , e então concluir que $x(t)$ apresenta o comportamento descrito pela Eq. 21). Nesta seção apresentamos como a observação do comportamento das condições de contorno no plano $\left(x^{2}, \dot{x}^{2}\right)$ (ou $\left.\left(E_{p}(t), E_{c}(t)\right)\right)$ pode ser empregado para aferir o comportamento assintótico para as soluções $x(t)$ encontradas para os casos do OHS e OHS amortecido, e como este comportamento é uma consequência das interações envolvidas, na próxima seção iremos considerar o caso da inclusão de um forçamento externo $F(t)$. 


\section{Caso incluindo o forçamento externo}

Nesta seção vamos considerar a situação da inclusão de um forçamento externo periódico da forma

$$
\frac{F(t)}{m}= \begin{cases}\frac{F_{0}}{m} \cos (\omega t), & t \geq t_{0} \\ 0 & , t \leq t_{0} .\end{cases}
$$

Empregaremos nesta seção a solução para osciladores harmônicos amortecidos e forçados descrita ao longo da seção 2, assim, na Eq. 11 iremos considerar $C_{ \pm}=1 \mathrm{e}$ $\gamma^{\prime}=2 \gamma=0.15$, bem como iremos escolher $\frac{F_{0}}{m}=\frac{1}{6.65}$, de forma a recuperar no assintótico o comportamento apresentado pela Figura 1(linha Azul). O comportamento descrito pela Eq. 23 pode ser representado por $\Theta(t-$ $\left.t_{0}\right) \frac{F_{0}}{m} \cos (\omega t)$, onde $\Theta\left(t-t_{0}\right)$ é a função de Heaviside 7

$$
\Theta(x-a)= \begin{cases}1, & x \geq a \\ 0, & x \leq a .\end{cases}
$$

Portanto, assumindo as Eqs.(8), (11) e (13), tendo em vista que a solução obtida com o método de operadores leva a soluções no plano complexo, na Figura 2 apresentamos no plano $\left(x^{2}, \dot{x}^{2}\right)$ o comportamento da solução numérica para $\operatorname{Re}\left(x^{2}(t)\right)$ e $\operatorname{Re}\left(\dot{x}^{2}(t)\right)$ para o intervalo onde $t \leq t_{0}$, que corresponde ao comportamento de um OHS amortecido.

A Figura 2 foi introduzida apenas como verificação da solução apresentada pelas Eqs. 8 8 13), considerando a inclusão do forçamento externo dado pela Eq. (23) temos o comportamento da solução numérica para $\operatorname{Re}\left(x^{2}(t)\right)$ e $\operatorname{Re}\left(\dot{x}^{2}(t)\right)$ para o intervalo $t \geq t_{0}$, como é possível verificar o comportamento da Figura 3 é oposto ao apresentado na Figura 2. Considerando um ciclo de períodos suficientemente grande (região assintótica) as linhas do

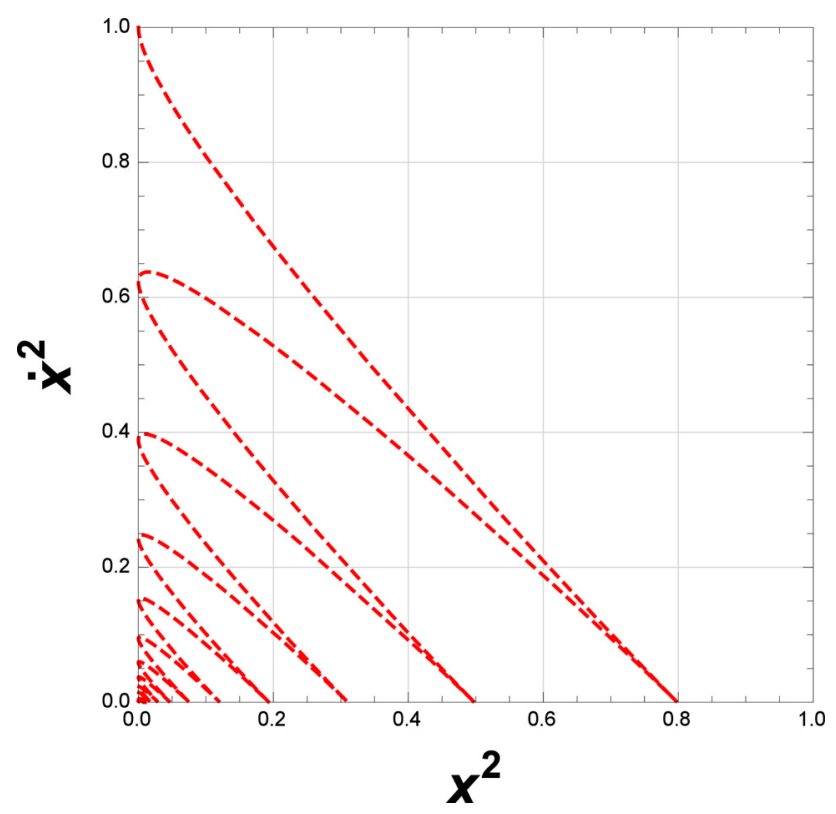

Figura 2: Comportamento no plano $\left(x^{2}, \dot{x}^{2}\right)$ para o OHS amortecido.

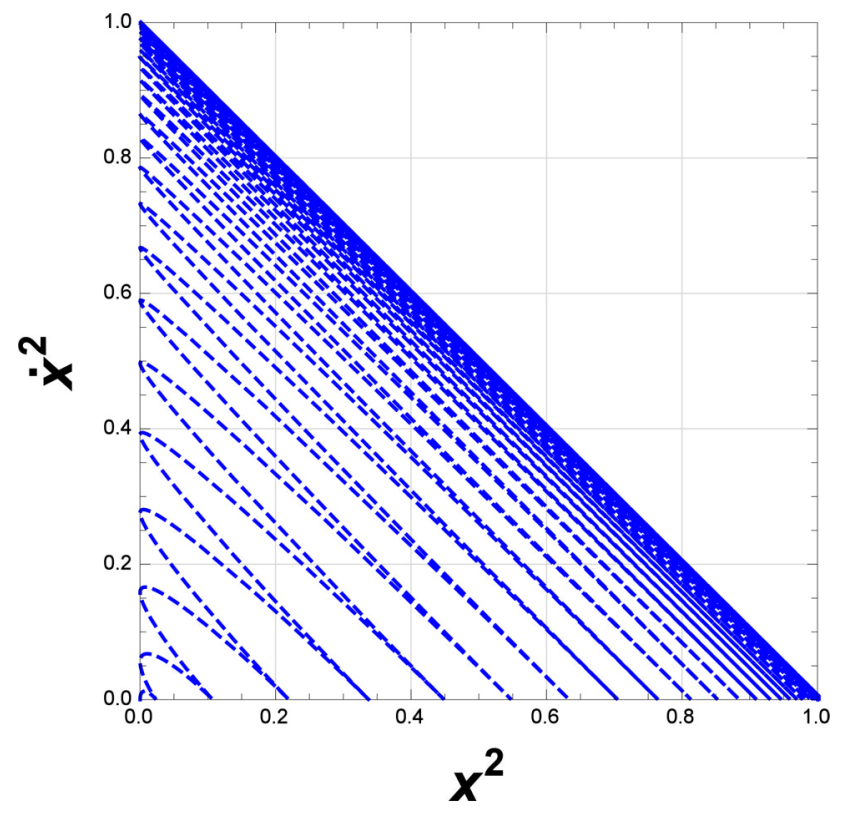

Figura 3: Comportamento no plano $\left(x^{2}, \dot{x}^{2}\right)$ para o OHS amortecido e forçado.

tracejado em azul descrito no gráfico tendem a se agrupar , de forma que no limite assintótico, no qual o forçamento externo superou a interação devido as forças de atrito, o sistema passa ao regime de um OHS análogo ao descrito na Figura 1. A combinação das Figuras 2 e 3 ilustra como a dinâmica do sistema OHS é modificada em frente a interações presentes em algum momento no intervalo de tempo considerado, então, assumindo a descrição apresentada na seção 2 é possível inferir a partir de uma análise do comportamento exibido na Figura 3 para a dinâmica exibida pelo sistema na regiâo assintótica $(n \rightarrow \infty)$

$$
\text { (Figura 3), }\left.x(t)\right|_{t \rightarrow n T} \sim x_{0} \cos (\omega t) \text {. }
$$

O tema tratado nas seções (2-4) já foi bastante discutido na literatura, o proposito de apresentação destas seções teve o intuito apenas de familiarizar o leitor com a discussão a respeito de como condições de contorno na região assintótica podem ser empregadas(e lidas) a partir da representação descrita nas Figuras (1-3). A fim de auxiliar na descrição do comportamento assintótico de sistemas como OHS e OHS amortecido e forçado, na seção seguinte iremos apresentar uma aplicação a sistemas físicos mais complexos, como exemplo iremos considerar o oscilador de Van der Pol 8]. Uma discussão mais detalhada e precisa a respeito do oscilador de Van der Pol e de sistemas não-lineares pode ser encontrada nas Refs. 911 .

\section{O Oscilador não-linear}

O oscilador conhecido como Oscilador de Van der Pol [8] foi originalmente proposto pelo Engenheiro Elétrico e Físico, Balthasar van der Pol em 1920 quando este 
estudava perturbações períódicas em um circuito com tubo de vácuo, como um tubo de neônio, sendo esta equação hoje em dia aplicada em vários contextos e situações, como a descrição de batimentos cardiacos [12]. A equação de Van der Pol é uma equação da forma de um OHS amortecido com um comportamento não-linear e que apresentamos abaixo

$$
\ddot{x}-\gamma\left(1-x^{2}\right) \dot{x}+x=0,
$$

o termo $\gamma\left(1-x^{2}\right)$ se comporta como um coeficiente de amortecimento não-linear, $\gamma(x)=\gamma\left(1-x^{2}\right)$, e o sinal (-) na frente sugere que este irá se comportar como um agente de forçamento externo, que pode ser comparado com o comportamento apresentado na Figura 3.

Na Figura 4 representamos o comportamento da solução obtida numericamente para a Eq. 26 no plano $\left(x^{2}, \dot{x}^{2}\right)$ para $\gamma=0.09$ (Figura a) e $\gamma=0.15$ (Figura b). Para o sistema dissipativo com forçamento periódico externo descrito ao longo da seção 4 , vimos que o comportamento assintótico deste sistema equivale ao comportamento de um OHS ( vide Figuras 3 e 1), uma vez que o forçamento externo periódico compensa a dissipação provocada pelo efeito do atrito. A fim de ganhar algum insight em relação a uma solução assintótica analítica para a Eq.(26), a exemplo da discussão apresentada ao longo da seção 3 onde a partir da Figura 1, inferimos o comportamento descrito pela Eq.21. De forma similar, nos parágrafos que seguem iremos traçar uma correspondência entre o comportamento descrito pelas Figuras 3 e 4. O comportamento assintótico descrito na Figura 4 corresponde no plano $\left(x^{2}, \dot{x}^{2}\right)$ a descrição de um ciclo limite [8, neste ponto devemos notar que para caracterização do ciclo limite representado, o comportamento não-linear do coeficiente de atrito irá funcionar como um forçamento externo. Considerando por exemplo o intervalo de tempo, $t_{1}=\frac{T}{4}$ e $t_{2} \approx 2 t_{1}$, com $t_{1}$ partindo do extremo a direita da linha verde continua representada na Figura 4b sobre a alça em vermelho, e $t_{2}$ o tempo gasto para percorrer o contorno até o mesmo ponto. $\mathrm{Na}$ Figura $4 \mathrm{~b}$, para um mesmo valor associado a $E_{p}$, verificase que temos valores distintos para $E_{c}(t)$ (em $t_{1}$ e $t_{2}$ ), o que não ocorre para um sistema conservativo como o representado na Figura 1.

Assim, a comparação na região assintótica entre as Figuras 3 e 4 nos permite propor que a existência da diferença entre $E_{c}\left(t_{1}\right)$ e $E_{c}\left(t_{2}\right)$, possa ser representada por uma diferença de fase e que provavelmente irá depender do valor assumido para o amortecimento $\gamma$ (vide Figura (4a)) e como $E_{c}(t)$ muda no tempo, portanto, é possível sugerir um "Anzatz"a partir da comparação na região assintótica entre as Figuras 3 e 4 , tal que

$$
\begin{aligned}
& x(t) \approx A \operatorname{Sin}\left(t+\frac{\gamma}{4} \dot{x}^{2}(t)\right) \\
& \dot{x}(t) \approx A \operatorname{Cos}(t) .
\end{aligned}
$$

O termo $\frac{\gamma}{4} \dot{x}^{2}(t)$ inserido na expressão acima é o responsável pela mudança no comportamento da Figura $4 \mathrm{~b}$
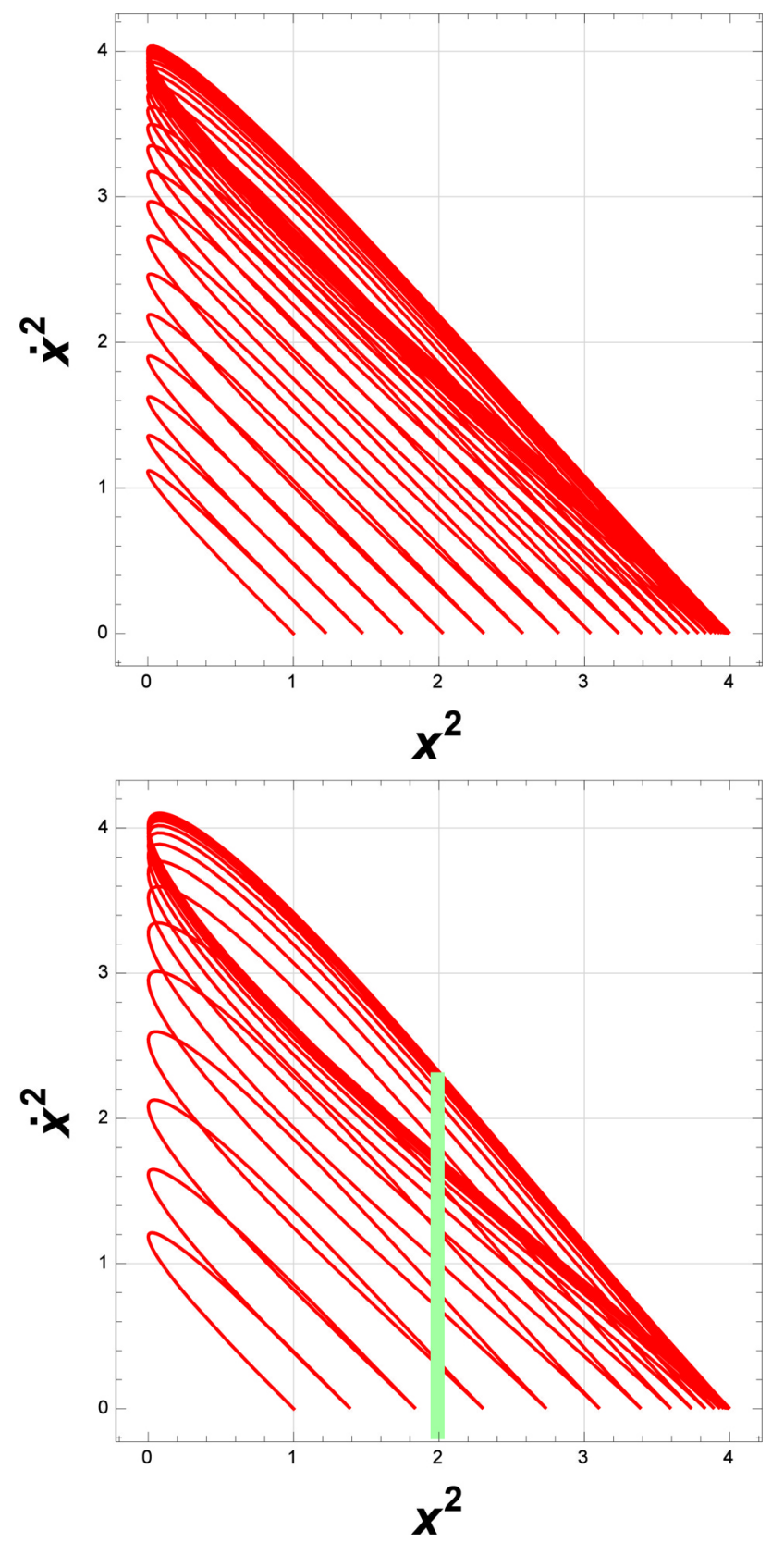

Figura 4: Comportamento no plano $\left(x^{2}, \dot{x}^{2}\right)$ para o de Van der Pol para (a) $\gamma=0.09$ e (b) $\gamma=0.15$

em relação a 3 , sendo que as expressões acima devem ser consideradas como uma descrição aproximada a ser empregada na região assintótica do tempo, os fatores $A$ e $\frac{1}{4}$, são ajustados de forma a fitar o comportamento do ciclo limite (região assintótica) para a equação de Van der Pol no plano $\left(E_{p}(t), E_{c}(t)\right)$ conforme representado na Figura 5 .

Assumindo $\gamma=0.15$, na Figura 5 apresentamos a superposição do comportamento da Eq.27) para $A=2$, com a solução numérica obtida para a equação de Van der Pol, com $(x(0)=1, \dot{x}(0)=0)$, onde a linha em vermelho correponde ao resultado para a equação de Van der Pol , e a linha azul pontilhada o obtido para Eq. 27). A 


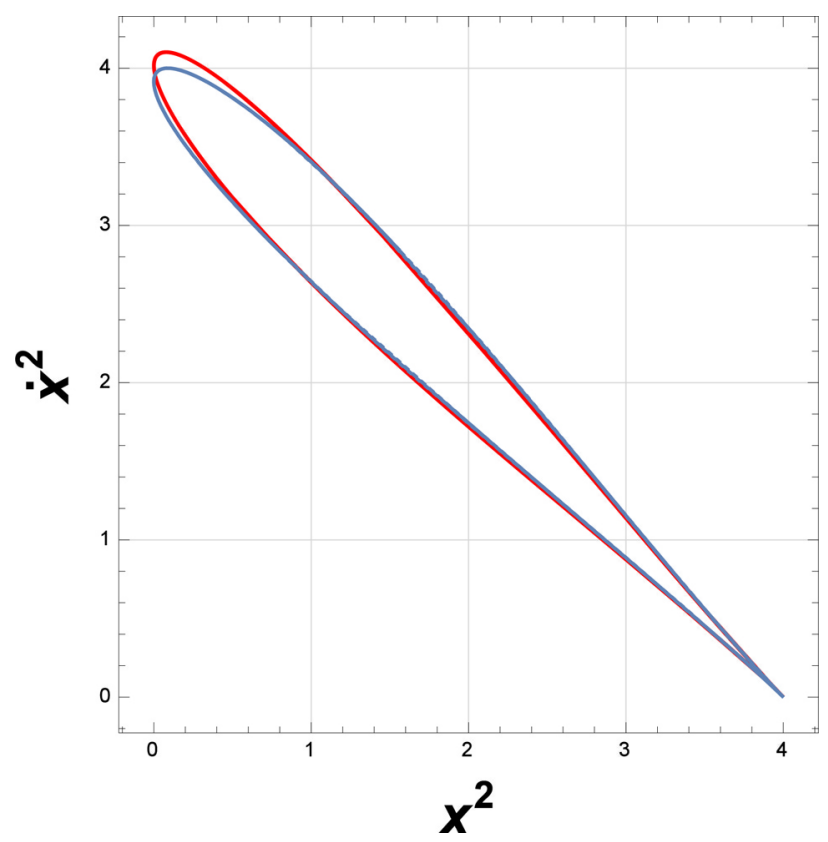

Figura 5: Comportamento do ciclo limite (região assintótica) para a equação de Van der Pol no plano $\left(x^{2}, \dot{x}^{2}\right)$

expressão Eq.(27) é o "Anzatz"proposto para a descrição do sistema na região onde $t \rightarrow n T$, para $n \rightarrow \infty$, que para uma consideração ingênua, $t \rightarrow \infty$, leva a

$$
\begin{aligned}
& x(t) \approx A \operatorname{Sin}(t) \\
& \dot{x}(t) \approx A \operatorname{Cos}(t),
\end{aligned}
$$

de forma que perdemos a informação a respeito do comportamento do termo $\frac{\gamma}{4} \dot{x}^{2}(t)$ no assintótico. A fim de contornar este problema devemos notar que para $\gamma=0$, $\operatorname{com} \omega_{0}^{2}=1$

$$
\dot{x}^{2}(t)+x^{2}(t)=A^{2} .
$$

Considerando a expansão em torno de $\gamma \approx 0$ na Eq.(27), ganhamos

$$
\dot{x}^{2}(t)\left(1+\frac{\gamma}{2} x(t) \dot{x}(t)\right)+x^{2}(t) \approx A^{2}+O\left(\gamma^{2}\right)
$$

de forma que na região assintótica, assumindo a previsão da Eq. 28) em conjunto com o resultado acima, temos a correção em $O(\gamma)$

$$
\begin{aligned}
& x(t) \approx A \operatorname{Sin}(t) \\
& \dot{x}(t) \approx A \operatorname{Cos}(t)\left(1+\frac{\gamma}{2} x(t) \dot{x}(t)\right)^{\frac{1}{2}}+O\left(\gamma^{2}\right) .
\end{aligned}
$$

A comparação do comportamento da equação acima com o resultante da Eq.(27) é apresentado na Figura 6 onde a linha pontilhada laranja representa o comportamento obtido para a Eq. 31. As expressões descritas pela Eq. (31) permitem de forma aproximada verificar que a equação de movimento em $O(\gamma)$ satisfeita é da forma

$$
\ddot{x}(t)+x(t) \approx \frac{A^{2} \gamma}{4} \dot{x}(t)\left(1-\frac{3}{2 A^{2}} x^{2}(t)\right)+O\left(\gamma^{2}\right),
$$

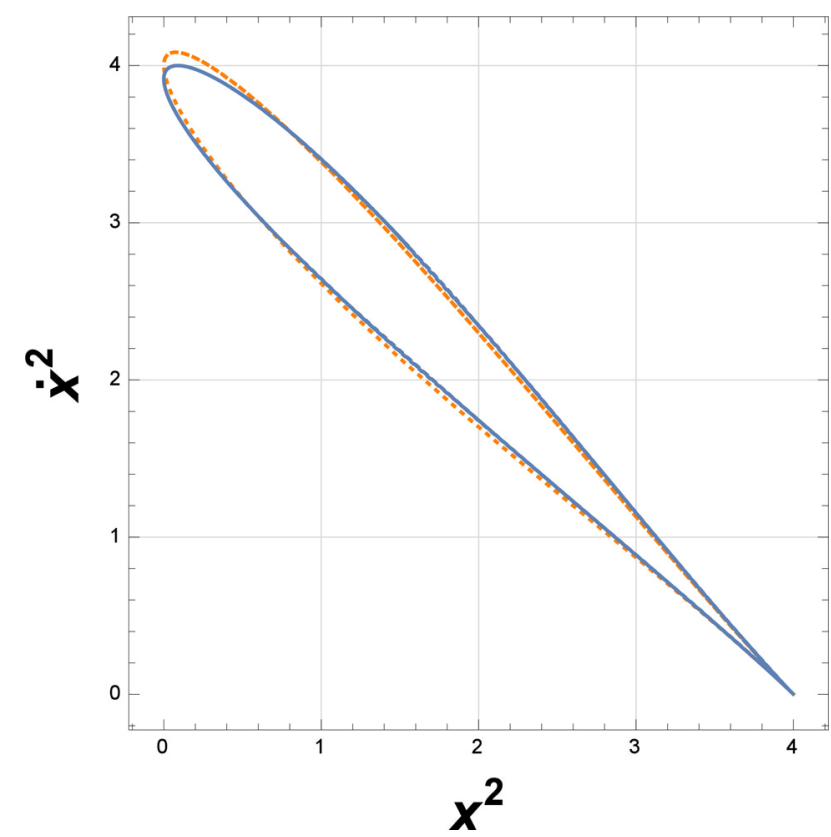

Figura 6: Comportamento do ciclo limite para a equação de Van der Pol para a solução assintótica, Eq.(31).

de maneira que esta corresponde a uma equação similar a Eq. 26) para $A=2$, onde neste caso o termo não linear é aproximado pelo coeficiente $\frac{3}{2 A^{2}} x^{2}(t)=\frac{3}{8} x^{2}(t)$.

\section{Conclusões}

Fenômenos oscilatórios são interesse em todos os campos da física e sistemas físicos que são governados por uma equação do tipo oscilador harmônico amortecido e forçado, como o circuito RLC submetido a uma tensão periódica. Neste trabalho apresentamos uma análise do comportamento assintótico de Osciladores Harmônicos Amortecidos e Forçados considerado a representação no espaço $\left(x^{2}, \dot{x}^{2}\right)$. Como vimos a abordagem assumida em tal plano mapeia o comportamento da energia total para uma partícula sujeita a um potencial na forma $a x^{2}(t)$, e como verificamos permite a avaliação mesmo de forma aproximada para sistemas não-lineares da evolução dinâmica de $x(t), \dot{x}(t)$ de forma independente na região assintótica do tempo. A forma particular de representação no espaço $\left(x^{2}(t), \dot{x}^{2}(t)\right)$ não é usualmente empregada na literatura, no sentido que abordagem convencional trata da representação de sistemas dinâmicos no espaço de face ordinário $(x(t), \dot{x}(t))$. Conforme discutimos ao final da seção 3, a Figura 1 permite a leitura e identificação direta para o comportamento assintótico para o OHS amortecido, e OHS amortecido com forçamento periódico, onde a atribuição considerada para o coeficiente de amortecimento foi apenas ilustrativa ( não focamos em algum sistema físico em específico). No tocante a aplicação descrita ao longo da seção 5 , não estamos discutindo implicações em específico, pois uma análise mais detalhada em relação ao comportamento analítico da solução da 
Eq.26 sairia do escopo deste trabalho, uma discussão adicional a respeito da solução da Eq. 26. pode ser encontrada nas Refs. [12], [13] [14]. Resumindo, verificamos que a partir da análise do comportamento assintótico das condições de contorno, tomando como exemplo o caso particular de Osciladores Harmônicos Amortecidos e Forçados, que é possível inferir o comportamento dinâmico nesta região para sistemas mais complexos, como o oscilador não-linear descrito na seção 5 .

\section{Agradecimentos}

Agradeço a A.A. Natale pela leitura e sugestões ao manuscrito, e ao Conselho Nacional de Desenvolvimento Científico e Tecnológico (CNPq) pelo apoio financeiro, processos no $302663 / 2016-9$ e 430466/2018-8.

\section{Referências}

[1] J. Figueira e A. Doff, Revista Brasileira de Ensino de Física 30, 1305 (2008).

[2] J. Figueira, J.C. Gentilini e A. Doff, Revista Brasileira de Ensino de Física 33, 1305 (2011).

[3] D. Halliday, R. Resnick e J. Walker, Fundamentos de Física (LTC, Rio de Janeiro, 2012), 9a ed., v. 2.

[4] F.W. Sears, M.W Zemansky, H.D. Young e R.A. Freedman, Física (Pearson Addison-Wesley, São Paulo, 2008), $12^{\mathrm{a}}$ ed., v. 2.

[5] P.A. Tipler e G. Mosca, Física: para cientistas e engenheiros (LTC, Rio de Janeiro, 2009), 6 $6^{\mathrm{a}}$ ed., v. 2.

[6] A.C. Bertuola, M.S. Hussein e M.P. Pato, Revista Brasileira de Ensino de Física 27, 327 (2005).

[7] E. Butkov, Física Matemática (LTC, Rio de Janeiro, 1988), $1^{\mathrm{a}}$ ed.

[8] P. Hagedorn, Oscilacões Não-Lineares (Edgard Blucher, São Paulo, 1984).

[9] J.K. Hale, Ordinary Differential Equations (Dover edition, New York, 2009).

[10] V. Arnold, Ordinary Differential Equations (SpringerVerlag, Heidelberg, 2006).

[11] F. Verhulst, Nonlinear Differential Equations and Dynamical Systems (Springer-Verlag, Heidelberg, 1990).

[12] R.L. Viana, Revista Brasileira de Ensino de Física 33, 3305 (2011).

[13] M.L. Cartwright e J.E. Littlewood, J. London Math. Soc. 20, 180 (1945).

[14] M.L. Cartwright, in: Contributions to the theory of nonlinear oscillations, editado por S. Lefschetz (Princeton University Press, Princeton, 1950), p. 149-241. 